\title{
Siloxane-PMMA hybrid anti-corrosion coatings reinforced by lignin
}

\author{
Samarah Vargas Harb, Bianca Machado Cerrutti, Sandra Helena Pulcinelli, \\ Celso Valentim Santilli, Peter Hammer*
}

Instituto de Química, UNESP-Univ Estadual Paulista, 14800-600 Araraquara, SP, Brazil

\section{A R T I C L E I N F O}

Available online 7 May 2015

\section{Keywords:}

Lignin

Siloxane-PMMA hybrid

Sol-gel process

Anti-corrosion coatings

\begin{abstract}
A B S T R A C T
Novel organic-inorganic hybrid coatings prepared using siloxane-poly(methyl methacrylate) (PMMA) were synthesized using the sol-gel process, with incorporation of lignin to improve mechanical strength, thermal stability and hydrophobicity, while maintaining the high anti-corrosive protection of siloxane-PMMA coatings. Dispersion of lignin in the siloxane-PMMA hybrid was analyzed by optical microscopy. Structural, morphological and wettability analysis of the about $2.5 \mu \mathrm{m}$ thick coatings, deposited on carbon steel by dip-coating, was performed by X-ray photoelectron spectroscopy, atomic force microscopy and contact angle measurements. Thermal stability was investigated by thermogravimetry and the mechanical properties were studied by microhardness and microscratch measurements. Electrochemical impedance spectroscopy was used to evaluate corrosion resistance in saline aqueous environments. All coatings showed good dispersion of lignin in the hybrid matrix and low surface roughness between 0.3 and $0.4 \mathrm{~nm}$. Incorporation of lignin increased hydrophobicity, microhardness and scratch resistance, in addition to shifting thermal degradation events to higher temperatures. The electrochemical tests showed that the hybrids act as efficient diffusion barriers, with corrosion resistance in the range of $10^{8} \Omega \mathrm{cm}^{2}$ after exposure to $3.5 \% \mathrm{NaCl}$ aqueous solution. The hybrid containing a intermediate lignin content of $0.10 \mathrm{wt}$ \% presented the best result, exhibiting the most hydrophobic surface ( $87.9^{\circ}$ water drop contact angle), the highest hardness value $(31.5 \mathrm{HV}$ ), high scratch resistance (up to $80.7 \mathrm{mN}$ ) and elevated impedance modulus $\left(10^{8} \Omega \mathrm{cm}^{2}\right)$ after 50 days exposure.
\end{abstract}

(C) 2015 Elsevier B.V. All rights reserved.

\section{Introduction}

The lignin macromolecule confers rigidity and impermeability to cell walls of terrestrial plants, usually corresponding to $15-30 \%$ of the dry weight of wood. As one of the most abundant biomacromolecules in nature, it is an important source of renewable biomass. It exhibits a three-dimensional network structure formed by phenylpropane units (Hydroxy phenyl-H, Syringyl-S, Guaiacyl-G) linked in more than 10 different ways, with the $\beta-0-4$ ether bond being one of the most frequent $[1,2]$. The lignin used in this study was extracted from sugar cane bagasse which contains 20-25\% lignin with predominantly syringyl units, G:S:H (1:2:0.8)-hardwood. Bagasse from sugar cane is derived from ethanol production; from the 532.75 million tons of sugar cane produced annually in Brazil, 26 to 76 million tons are bagasse, from which approximately 7.5 million tons of lignin are generated [3]. Biodiesel production also generates residual lignocellulosic material (straw, trunk, branches, bark and mulch) from oilseed species such as soybean, castor and cotton [3]. In the USA alone about 50 million

\footnotetext{
* Corresponding author at: Instituto de Química, Departamento de Físico-Química, Rua Prof. Francisco Degni, 55, 14800-060 Araraquara, SP, Brazil. Tel.: + 551633019887.

E-mail addresses: samarah.v.h@hotmail.com (S.V.Harb), bicerr@gmail.com (B.M. Cerrutti), sandrap@iq.unesp.br (S.H. Pulcinelli), santilli@iq.unesp.br (C.V. Santilli), peter@iq.unesp.br (P. Hammer).
}

tons of lignin are produced each year [2,4], most of which are burnt to generate energy.

However, more noble lignin applications have been found to add value to the waste, as basic material for aromatic (phenolic) compounds [2], reinforcement of materials [5,6], stabilizer for polymers [7], production of sensors [8], fiberboards [9], polyurethane foams [10] and wood adhesive for constructing particleboard [11,12], among others. Properties such as low density [6], low abrasive character [6] and hydrophobicity [1] in addition to the low cost make lignin ideal to use as additive in organic-inorganic hybrids for anti-corrosion coatings. Moreover, its phenolic nature is expected to increase resistance of the polymeric phase to oxidation, radiation and exposure to elevated temperatures $[2,3,13]$.

The siloxane-PMMA system is an interesting organic-inorganic hybrid material due to its transparency, chemical stability and wide range of applications. PMMA is rigid, transparent, and colorless thermoplastic, also known as Plexiglas and acrylic, used extensively in everyday life. The inclusion of an inorganic component such as silica into the PMMA matrix substantially improves its mechanical properties, thermal stability and adhesion to metallic substrates $[14,15]$. Recent studies showed that siloxane-PMMA coatings deposited on steel exhibit excellent corrosion resistance both in saline and in saline/acidic environments. Detailed analyses indicated that the inorganic phase has an important role in promoting adhesion between the coating and the 

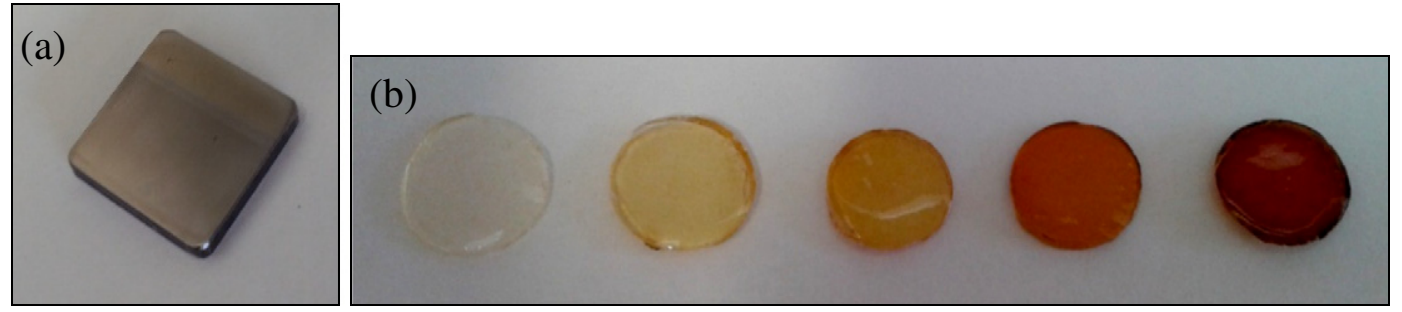

Fig. 1. Image of (a) coated carbon steel and (b) siloxane-PMMA monoliths with increasing lignin content: $0.00,0.05,0.100 .50$ and 1.00 wt.\%.

metal substrate, while the organic phase hermetically seals the film structure. Thus the ramified siloxane cross-link nodes interconnected covalently by short polymeric chains form a chemically inert barrier, which prevents penetration of species that initiate corrosive processes $[16,17]$. Furthermore, the incorporation of additives such as cerium ions as corrosion inhibitor and carbon nanotubes results in improvement of the corrosion protection efficiency and mechanic stability of the hybrids $[18,19,20]$.

Damages caused by corrosion are responsible for huge financial losses in industries, lack of safety in equipment and environmental contamination. Since it is not possible to prevent metal corrosion completely, new methodologies with special alloys, corrosion inhibitors and protective films have been produced to delay the corrosion processes for increasing time periods [17]. New materials that act as barriers against diffusion of aggressive species have been widely investigated, especially due to the need of replacing corrosion inhibitors based on chromium. Such inhibitors are largely used to prevent metallic corrosion, but they cause damage to health and environment [21].

In this work we focus on the possibility to mechanically reinforce the siloxane-PMMA hybrids and improve their thermal stability by incorporation of increasing amounts of lignin, maintaining the excellent anti-corrosion performance of the material. For this purpose we studied the structural, thermal and mechanical properties of hybrid materials by X-ray photoelectron spectroscopy (XPS), atomic force microscopy (AFM), contact angle, thermogravimetry, microhardness and microscratch measurements. The electrochemical performance of the coatings in standard saline solution was studied using electrochemical impedance spectroscopy (EIS).

\section{Materials and methods}

Lignin supplied by Dedini SA (Piracicaba, São Paulo, Brazil) was extracted using the organosolv process (DHR-Dedini Rapid Hydrolysis) with ethanol/water as solvent $[22,23]$. The powder lignin was washed with water at $70{ }^{\circ} \mathrm{C}$ for 30 min under mechanical stirring to remove residual sugars and impurities, filtered and dried at $105{ }^{\circ} \mathrm{C}$ until constant weight [24]. The following commercially available reagents were used as received: 3-methacryloxy propyltrimethoxy-silane (MPTS, Sigma-Aldrich), benzoyl peroxide (BPO, Sigma-Aldrich), tetraethylorthosilicate (TEOS, Sigma-Aldrich), ethanol (Sigma-Aldrich), nitric acid (Sigma-Aldrich), and tetrahydrofuran (THF, Sigma-Aldrich). Methyl methacrylate (MMA, Sigma-Aldrich) was distilled to remove inhibitors of polymerization.

The siloxane-PMMA hybrids were prepared in three steps, as reported previously [17]: (1) radical copolymerization of MMA with MPTS using BPO as thermal initiator and THF as solvent, (2) acid catalyzed hydrolysis and condensation of TEOS in ethanol medium, using the sol-gel process, and (3) mixture of the organic and inorganic phases. MPTS is composed by a siloxane and a methacrylate group that act as a coupling agent between the organic and inorganic phase. Lignin was added into the inorganic phase, step (2), in concentrations: $0.00 \mathrm{wt} . \%$, $0.05 \mathrm{wt} . \%, 0.10 \mathrm{wt} . \%, 0.50 \mathrm{wt} . \%$, and $1.00 \mathrm{wt} . \%$, relative the mass of the hybrid. All molar ratios were kept constant: MMA/MPTS $=8$, MMA/ TEOS $=4, \mathrm{BPO} / \mathrm{MMA}=0.01, \mathrm{H}_{2} \mathrm{O} / \mathrm{Si}=3.5$ and ethanol $/ \mathrm{H}_{2} \mathrm{O}=0.5$ [17]. The hybrids are referred as Ligx.xx\% where $\mathrm{x} . \mathrm{xx}$ is the lignin concentration in the siloxane-PMMA matrix. The homogeneous, transparent hybrids were deposited on carbon steel by dip-coating and dried on Teflon dishes to obtain monoliths. The carbon steel A1010 substrates (25 $\mathrm{mm} \times 25 \mathrm{~mm} \times 5 \mathrm{~mm}$ ) with a nominal composition (wt.\%) of $\mathrm{C}=0.15 \%, \mathrm{Mn}=0.69 \%, \mathrm{Si}=0.13, \mathrm{P}=0.031 \%$ and $\mathrm{S}=0.010 \%$, with the balance of Fe, were polished using an AROTEC polisher with silicon carbide paper grades 100, 320, 600 and 1500, and cleaned with isopropyl alcohol. Dip-coating was used to deposit the hybrid coatings, applying 3 dips at a withdraw rate of $14 \mathrm{~cm} \mathrm{~min}^{-1}$. Subsequent drying $\left(60^{\circ} \mathrm{C}\right.$ for $24 \mathrm{~h}$ ) and thermal cure $\left(160{ }^{\circ} \mathrm{C}\right.$ for $\left.180 \mathrm{~min}\right)$ ensured a high degree of polymerization of the siloxane-PMMA hybrid structure.

EIS, XPS, AFM, contact angle, microscratch, pull-off and thickness measurements were performed on coated carbon steel. Analyses by optical microscopy, microhardness and thermogravimetry were performed on monoliths (Fig. 1). An Olympus Microscope model Bx41 was used to evaluate the lignin dispersion in the hybrids. The RMS surface roughness was determined using topography images with an area of $1 \mu \mathrm{m}^{2}$, obtained with an Agilent 5500 Atomic Force Microscope (AFM) in the tapping mode. The coating thickness was determined
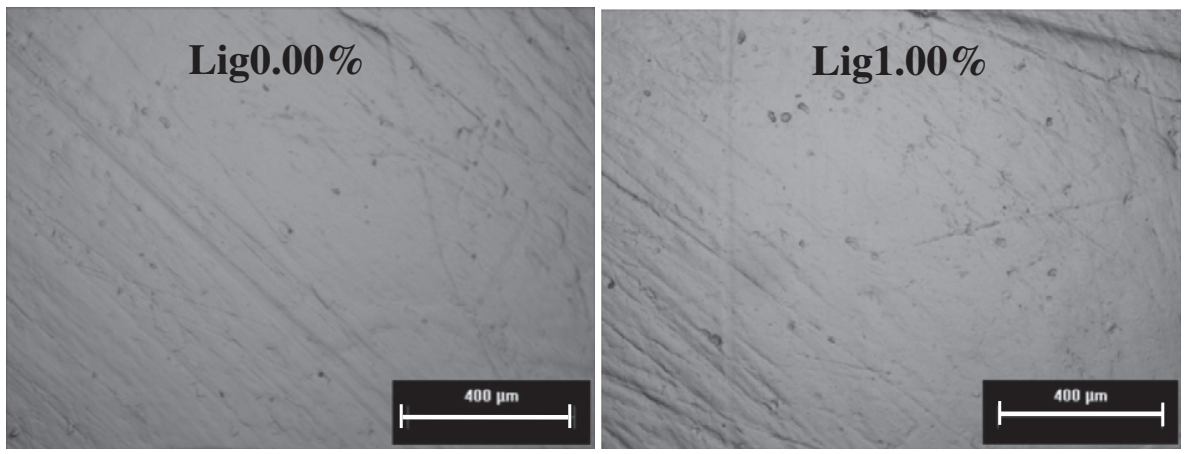

Fig. 2. Optical microscopy of siloxane-PMMA monoliths without lignin and with the highest lignin concentration (1.00 wt.\%). 

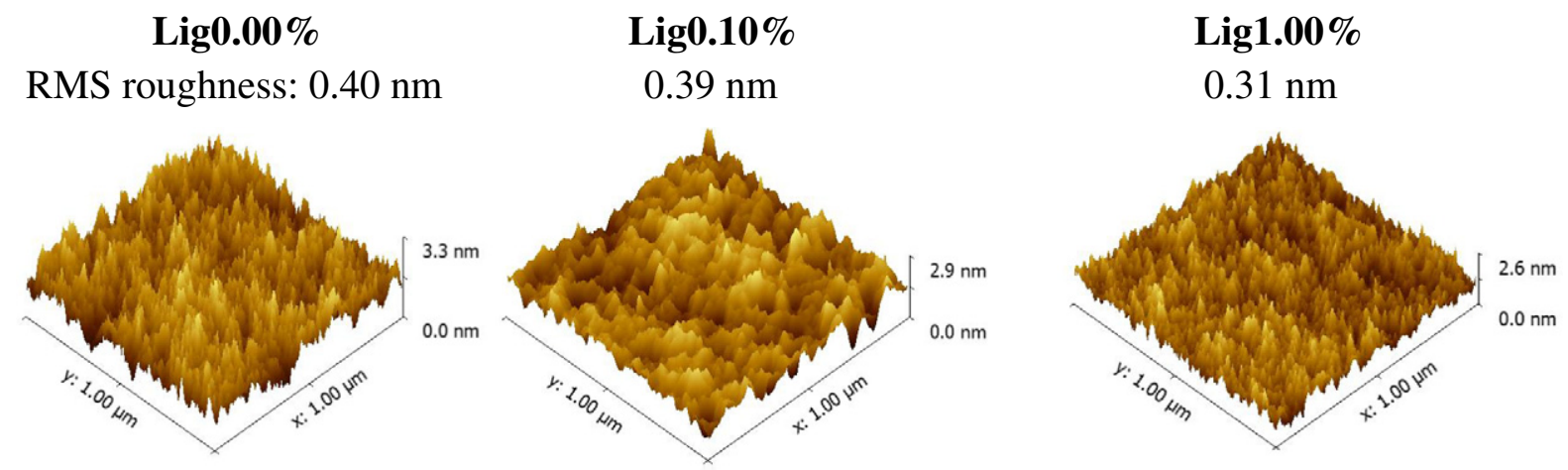

Fig. 3. AFM topography images and the calculated RMS roughness of siloxane-PMMA hybrids loaded with different quantities of lignin.

using Filmetrics F3-CS optical system. The adhesion of the coatings to the steel surface was evaluated by the ASTM D4541 method using a Posi-Test Pull-Off Adhesion Tester (De Felsko), determining the tensile pull-off force of detaching.

X-ray photoelectron spectroscopy (XPS) analysis of lignin and hybrids containing lignin was carried out in a UNI-SPECS UHV Surface Analysis System, using a pressure of less than $10^{-7} \mathrm{~Pa}$, with $\mathrm{Mg} \mathrm{K} \alpha$ radiation $(\mathrm{h} v=1253.6 \mathrm{eV})$ for excitation. The high resolution spectra were measured with pass energy of $10 \mathrm{eV}$. The inelastic background of the photoemission peaks of $\mathrm{C} 1 \mathrm{~s}, \mathrm{O} 1 \mathrm{~s}$ and $\mathrm{Si} 2 \mathrm{p}$ was subtracted using Shirley's method. The composition of the surface region $(<5 \mathrm{~nm})$ was determined from the ratio of the peak areas corrected by the sensitivity factors of the corresponding elements. Due to sample charging, the binding energy scale was corrected using the $C 1$ s hydrocarbon component, fixed at $285.0 \mathrm{eV}$. For analysis of the local structure, the spectra were deconvoluted using Voigt functions.

Thermogravimetry measurements of the hybrids were undertaken in a TA Instruments SDT Q600 equipment, using nitrogen atmosphere with continuous flow of $70 \mathrm{~mL} \mathrm{~min}{ }^{-1}$, sample mass of $7.0 \mathrm{mg}$ in an alumina crucible at a heating rate of $10^{\circ} \mathrm{C} \mathrm{min}^{-1}$.

The contact angles between the coated steel and water sessile drop were measured using a Contact Angle System OCA, DATAPHYSICS, coupled with a CCD camera. Each contact angle was obtained from an average of 5 measurements, using $7 \mu \mathrm{L}$ of distilled water.

The hardness measurements were performed in a Buehler Micromet 2003 Microhardness Tester using a load of $0.5 \mathrm{kgf}$. A diamond tip penetrates the sample at a given force and the diagonals of the rhombus imprinted mark are measured by a microscope. The average of these two measurements is used to determine the Vickers hardness, HV [25]. Each hardness value was calculated from an average of 5 hardness measurements with a standard deviation of less than $10 \%$.

The microscratch measurements were undertaken in homemade equipment at the National Physical Laboratory (London) to evaluate scratch resistance and adherence of the coatings. For each sample, 3 tracks $6 \mathrm{~mm}$ long were performed using an increasing load (from $2 \mathrm{mN}$ to $100 \mathrm{mN}$ ), with a diamond tip with spherical conical geometry
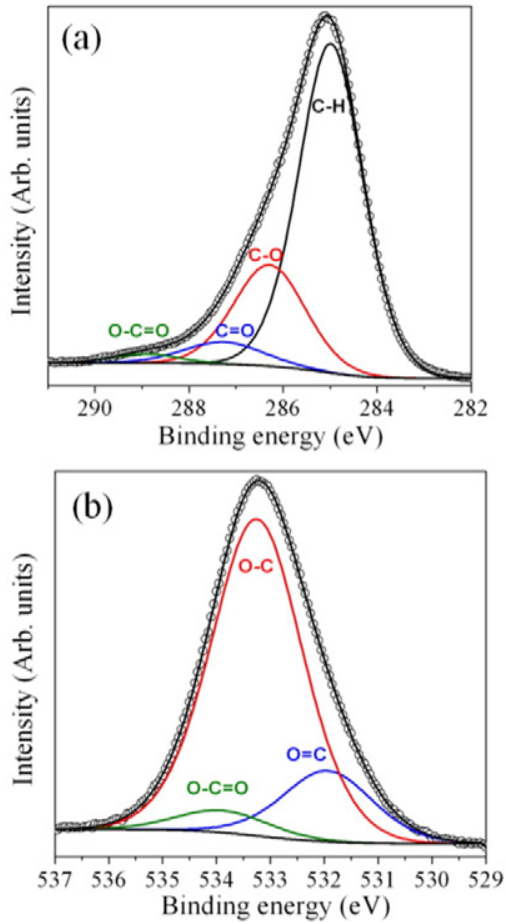

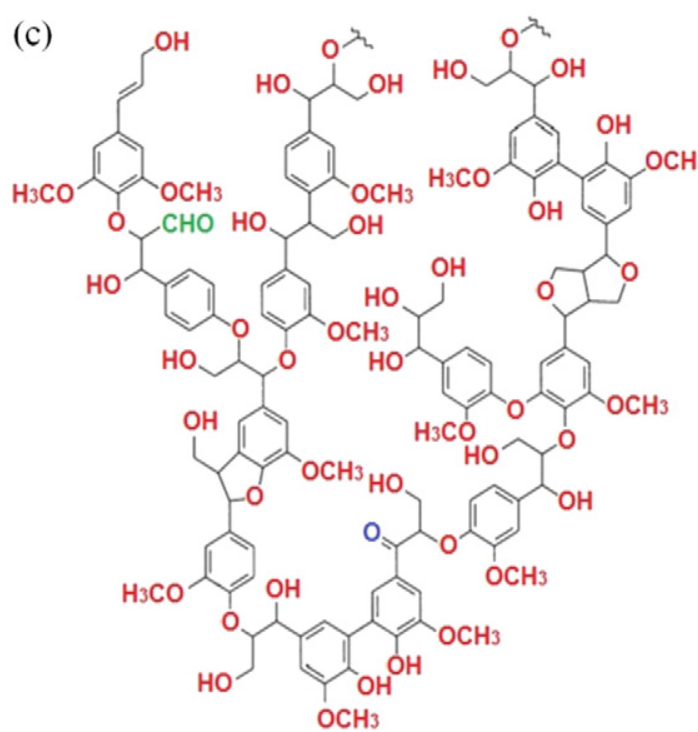

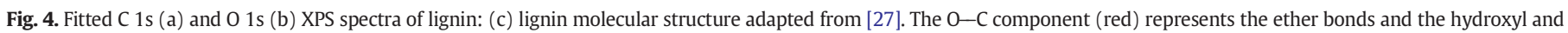
methyl groups. 

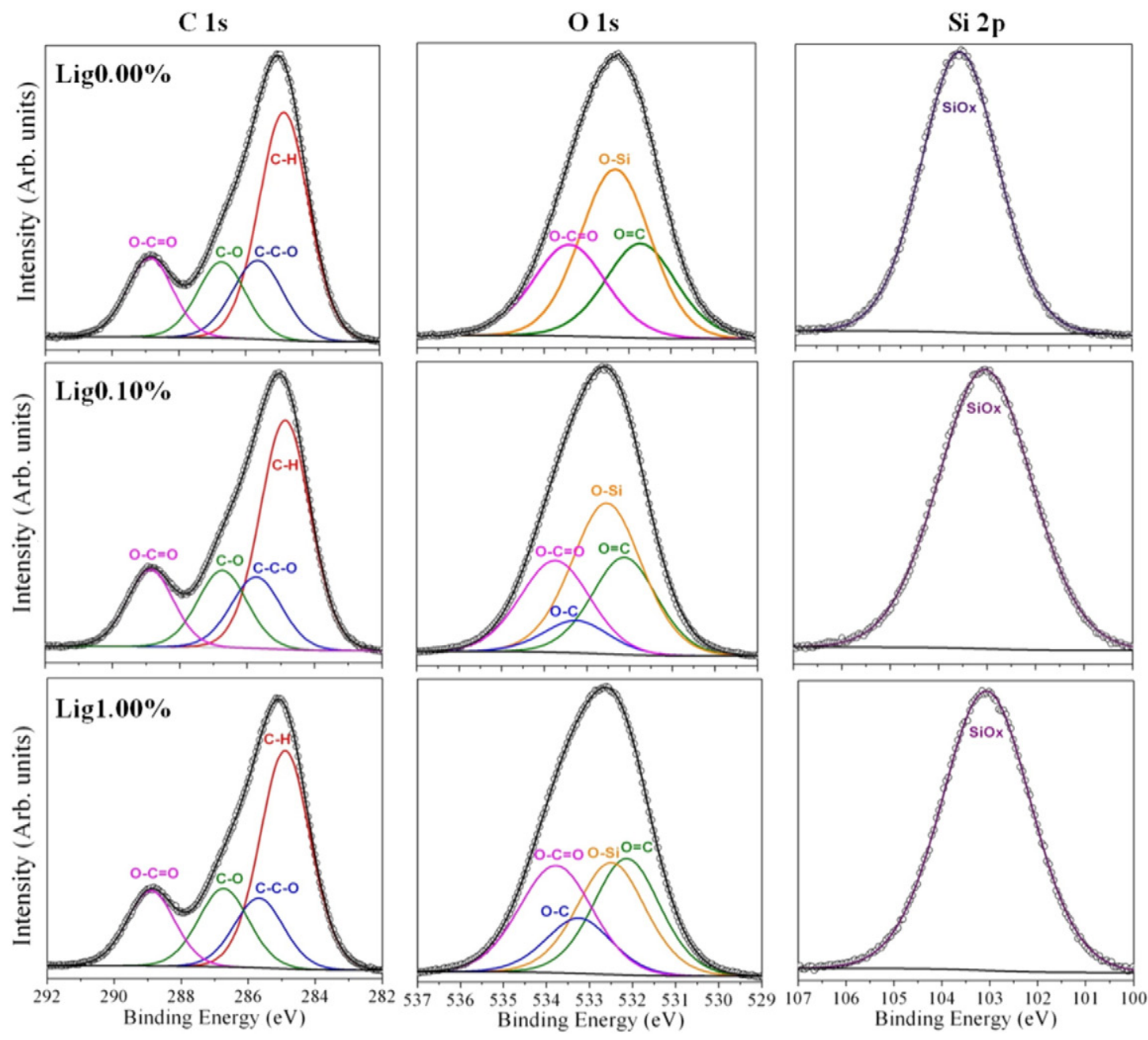

Fig. 5. Fitted C 1s, O 1s and Si 2p XPS spectra of the siloxane-PMMA hybrids (structure inset) containing $0.00,0.10$ and 1.00 wt.\% of lignin.

and $10 \mu \mathrm{m}$ radius. The equipment provides the friction coefficient with the track distance. The results were analyzed using a Nikon Measuring Microscope MM-60, coupled with a Nikon SC-213 Digital Counter, from which it was possible to measure the critical force at which delamination occurs, using:

Breaking Force $(F b)=$ Fstart $+($ Fend-Fstart $) x\left(\frac{L-l 2}{L}\right)$

where Fstart is the initial force $(2 \mathrm{mN})$, Fend is the final force $(100 \mathrm{mN})$, L is the track length $(6 \mathrm{~mm})$, and $\mathrm{l}_{2}$ is the distance between the track end and the first point of delamination, which was measured with the optical microscope.

The corrosion protection efficiency of coated carbon steel and of the bare carbon steel surface was evaluated using electrochemical impedance spectroscopy (EIS). These measurements were carried out at $25{ }^{\circ} \mathrm{C}$ with $80 \mathrm{~mL}$ of a neutral aqueous solution of $3.5 \% \mathrm{NaCl}$, applying a sinusoidal potential perturbation of $10 \mathrm{mV}$ (rms) after checking Eoc for $5 \mathrm{~min}$, once a week. The scanning frequency ranged from $10^{5}$ to $10^{-3} \mathrm{~Hz}$ with 10 points/decade using a Potentiostat/Galvanostat EG\&G Parc-273 and a Frequency Response Analyzer Solartron-SI1255 impedance module. $\mathrm{An} \mathrm{Ag} / \mathrm{AgCl} / \mathrm{KCl}_{\text {sat }}$ electrode was used as reference, connected to the working solution through a Luggin capillary, a Pt grid was used as the auxiliary electrode. Also used were a fourth platinum electrode and a capacitor ( $10 \mu \mathrm{F})$ between the fourth and the reference electrodes. The sample area exposed to the saline solution was $1 \mathrm{~cm}^{2}$.

\section{Results and discussion}

\subsection{Structural and thermal features}

The hybrid homogeneity depends on the compatibility between the siloxane-PMMA matrix and lignin biopolymer, being essential for anticorrosive action and reinforcing effect [26]. PMMA and siloxane are polar materials that are compatible with the lignin polar structure (average 1-2 hydroxyl groups per monomer) used in this work, yielding a compatible blending in the liquid state, which should flavor the

Table 1

Atomic percentage of the element constituents of the siloxane-PMMA hybrids loaded with different quantities of lignin.

\begin{tabular}{lccc}
\hline & Lig0.00\% & $\begin{array}{l}\text { Lig0.10\% } \\
\text { (at.\%) }\end{array}$ & Lig1.00\% \\
\hline Carbon & 56.6 & 56.7 & 60.9 \\
Oxygen & 34.9 & 35.2 & 33.6 \\
Silicon & 8.5 & 8.1 & 6.1 \\
C/Si & 6.6 & 7.0 & 9.9 \\
\hline
\end{tabular}

Experimental error: $\pm 5 \%$. 


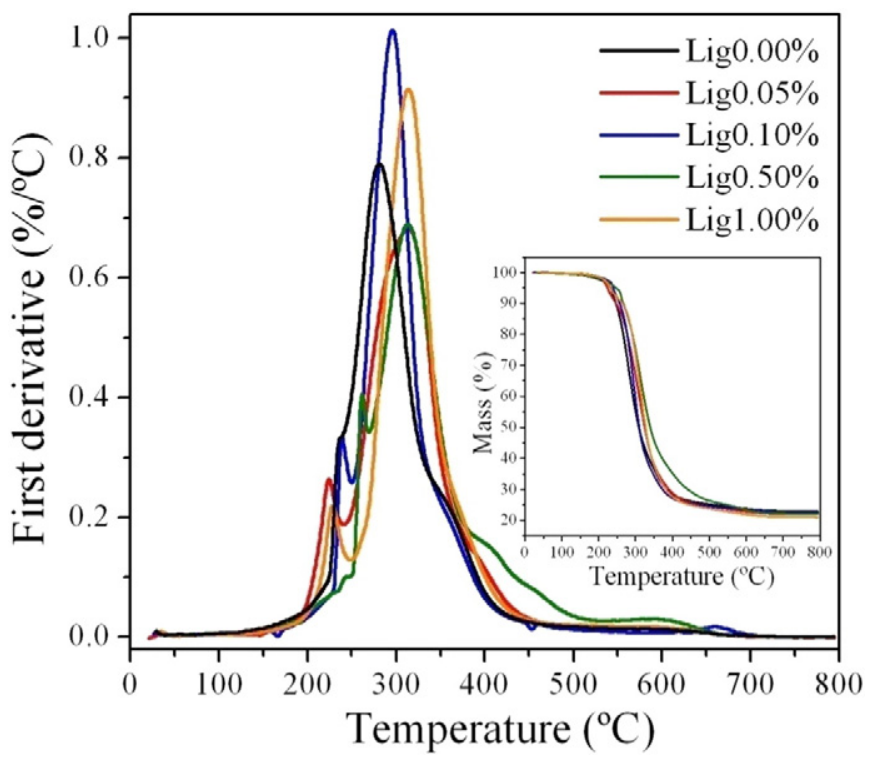

Fig. 6. DTG and TG curves (inset) of siloxane-PMMA hybrids with increasing lignin content.

formation of a highly homogeneous hybrid material. As shown by optical micrograph, displayed in Fig. 2, irrespective of lignin loading quantities all samples showed a homogenous structure without microscopic segregations. No lignin agglomerates were observed, which could impair the high anticorrosive efficiency of the siloxane-PMMA coating and the mechanical reinforcement. The AFM topography images in Fig. 3 confirmed a low surface roughness $\left(R_{\text {RMS }}<0.40 \mathrm{~nm}\right)$ and excellent lignin dispersion in the siloxanePMMA hybrid matrix.

Quantitative XPS analysis was used to determine the atomic composition of the lignin reference, containing $81 \pm 4$ at.\% of carbon and $19 \pm 1$ at.\% of oxygen. The fitted $C$ 1s spectra in Fig. 4-a showed an intense component related to $\mathrm{C}-\mathrm{H}$ bonds, in addition to $\mathrm{C}-\mathrm{O}, \mathrm{C}=\mathrm{O}$ and $\mathrm{O}-\mathrm{C}=\mathrm{O}$ bonds. Three components were identified in the fitted O1s spectra (Fig. 4-b), assigned to $\mathrm{O}-\mathrm{C}, \mathrm{O}-\mathrm{C}=\mathrm{O}$ and $\mathrm{O}=\mathrm{C}$ bonds. The proportion of these bonds is consistent with that reported for the lignin structure (Fig. 4-c), including hydroxyl, methoxyl, carbonyl and carboxyl functionalities and ether bonds [12].

The deconvoluted C 1s, 0 1s and Si 2p XPS core-level spectra allowed studying the evolution of the local bonding structure with increasing lignin loading (Fig. 5). The chemical environments of the molecular structure of the pure siloxane-PMMA hybrid were identified in the $\mathrm{C}$ 1s spectra, showing the expected proportion of intensities associated with $\mathrm{C}-\mathrm{H}, \mathrm{C}-\mathrm{C}-\mathrm{O}, \mathrm{C}-\mathrm{O}$ and $\mathrm{O}-\mathrm{C}=\mathrm{O}$ groups. For the $\mathrm{O} 1 \mathrm{~s}$ spectra three components were fitted, two related to $\mathrm{O}-\mathrm{C}=\mathrm{O}$ and $\mathrm{O}=\mathrm{C}$ bond of PMMA and MPTS, and one associated with $\mathrm{O}-\mathrm{Si}$ bond of the inorganic network. With addition of lignin content a fourth component

Table 2

Main thermal decomposition events, coating thickness and detaching force of hybrids, containing increasing fraction of lignin (see text).

\begin{tabular}{llllll}
\hline Sample & $\begin{array}{l}\mathrm{T}_{1} \\
\left({ }^{\circ} \mathrm{C}\right)\end{array}$ & $\begin{array}{l}\mathrm{T}_{2} \\
\left({ }^{\circ} \mathrm{C}\right)\end{array}$ & $\begin{array}{l}\text { Residue } \\
(\%)\end{array}$ & $\begin{array}{l}\text { Coating } \\
\text { thickness }(\mu \mathrm{m})\end{array}$ & $\begin{array}{l}\text { Detaching } \\
\text { force }(\mathrm{MPa})\end{array}$ \\
\hline Lig0.00\% & 235 & 280 & 22.9 & 2.35 & 3.6 \\
Lig0.05\% & 225 & 315 & 22.1 & 2.59 & 3.7 \\
Lig0.10\% & 240 & 295 & 22.4 & 2.52 & 4.1 \\
Lig0.50\% & 260 & 315 & 22.1 & 2.68 & 3.7 \\
Lig1.00\% & 230 & 315 & 21.1 & 2.65 & 3.4 \\
\hline
\end{tabular}

$\mathrm{T}_{1}$ : temperature of maximum degradation rate of the first event.

$\mathrm{T}_{2}$ : temperature of maximum degradation rate of the second event.

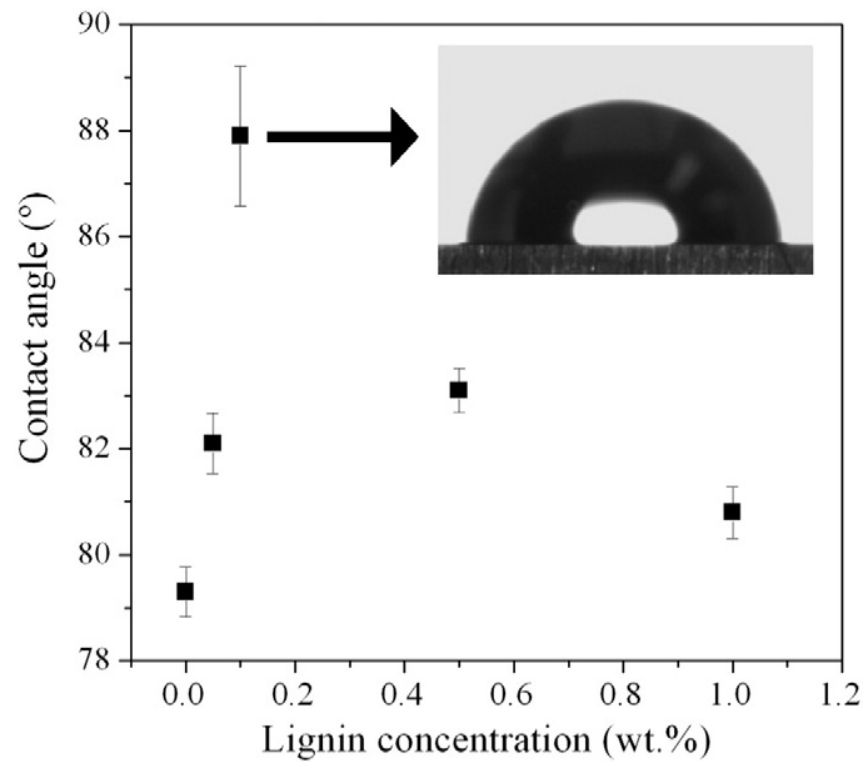

Fig. 7. Contact angle between the siloxane-PMMA hybrid surface and water as a function of the lignin fraction.

appeared related to $\mathrm{O}-\mathrm{C}$ bond due to ether groups present in lignin structure. The inorganic network stoichiometry, $\mathrm{SiO}_{\mathrm{x}}(1.5<\mathrm{x}<2)$, determined by the binding energy of the Si $2 \mathrm{p}$ peak indicates the presence of $\mathrm{Si}-\mathrm{O}-\mathrm{Si}$ and $\mathrm{Si}-\mathrm{OH}$ groups [28].

The elemental composition of the samples, derived from the quantitative analysis, is listed in Table 1 . The data confirmed the presence of lignin in the hybrid network, evidenced by the increasing $\mathrm{C} / \mathrm{Si}$ ratio coming from to the contribution of the additional carbon of the lignin structure $(81.4 \%)$.

The thermogravimetric curves, displayed in Fig. 6, indicate that the siloxane-PMMA hybrids are thermally stable up to $150{ }^{\circ} \mathrm{C}$, at which the organic phase starts to degrade. The differential themogravimetric curves showed that PMMA depolymerization under nitrogen occurs in two steps: the first with maximum near to $230{ }^{\circ} \mathrm{C}$ is due to scission of

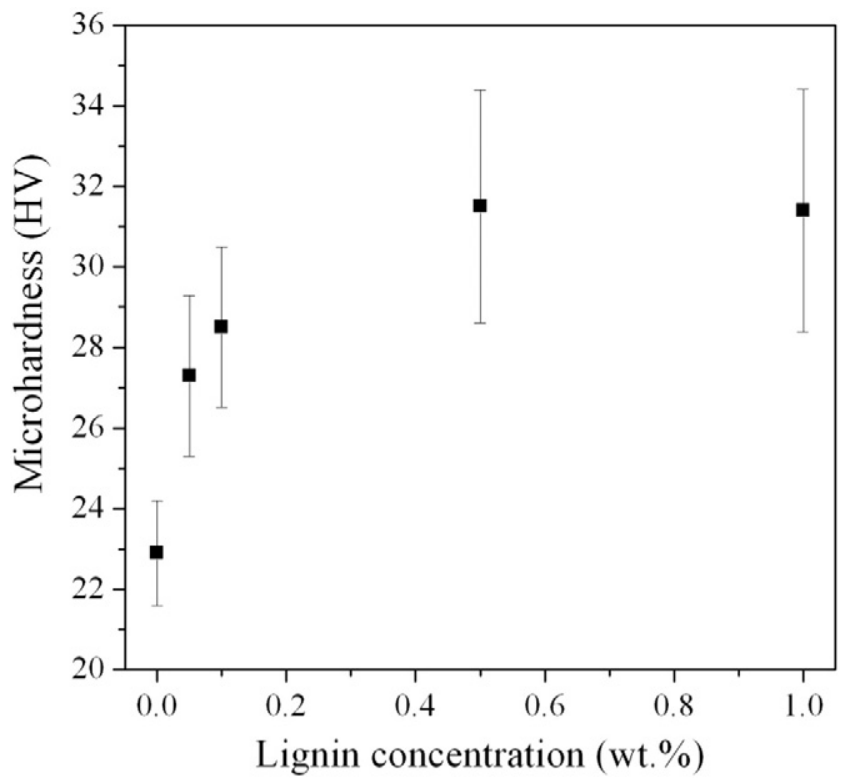

Fig. 8. Microhardness of the siloxane-PMMA hybrid monoliths as a function of the lignin fraction. 


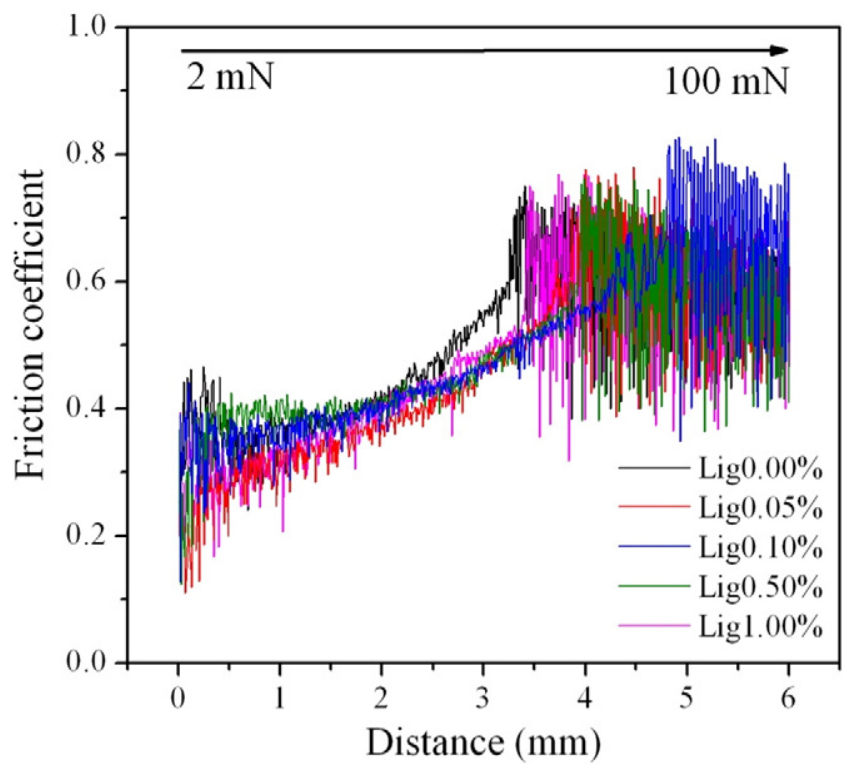

Fig. 9. Scratch test friction coefficient as a function of increasing load for hybrid coatings containing increasing lignin content.

weak head-to-head linkages and unsaturated vinylidene ends, while the second above $300{ }^{\circ} \mathrm{C}$ is associated with random chain scission [29, 30]. The uncompleted condensed inorganic network dehydrates at high temperatures $\left(>350^{\circ} \mathrm{C}\right)$ and forms stoichiometric silica (residue) [31]. The addition of lignin shifted degradation to higher temperatures,

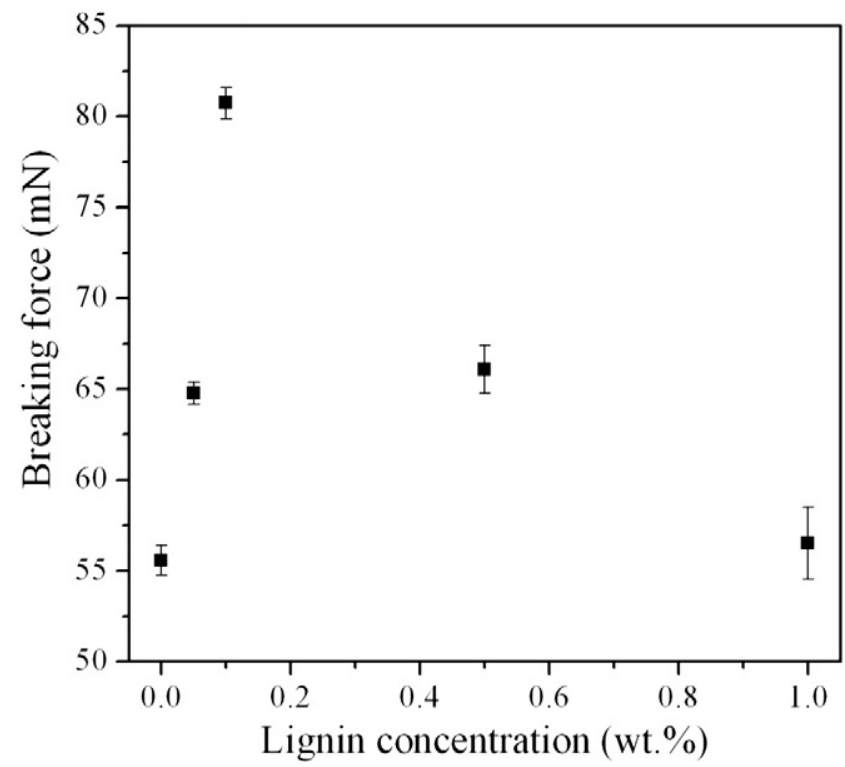

Fig. 11. Critical delamination force of siloxane-PMMA hybrid coatings on carbon steel as a function of the lignin content.

as shown in Table 2. This can be ascribed to the ability of lignin to trap radicals formed in depolymerization, thus enhancing thermal stability. The lignin phenolic structure can act as anti-oxidant and UV stabilizer for polymers scavenging radicals introduced by irradiation or heating $[12,32]$.

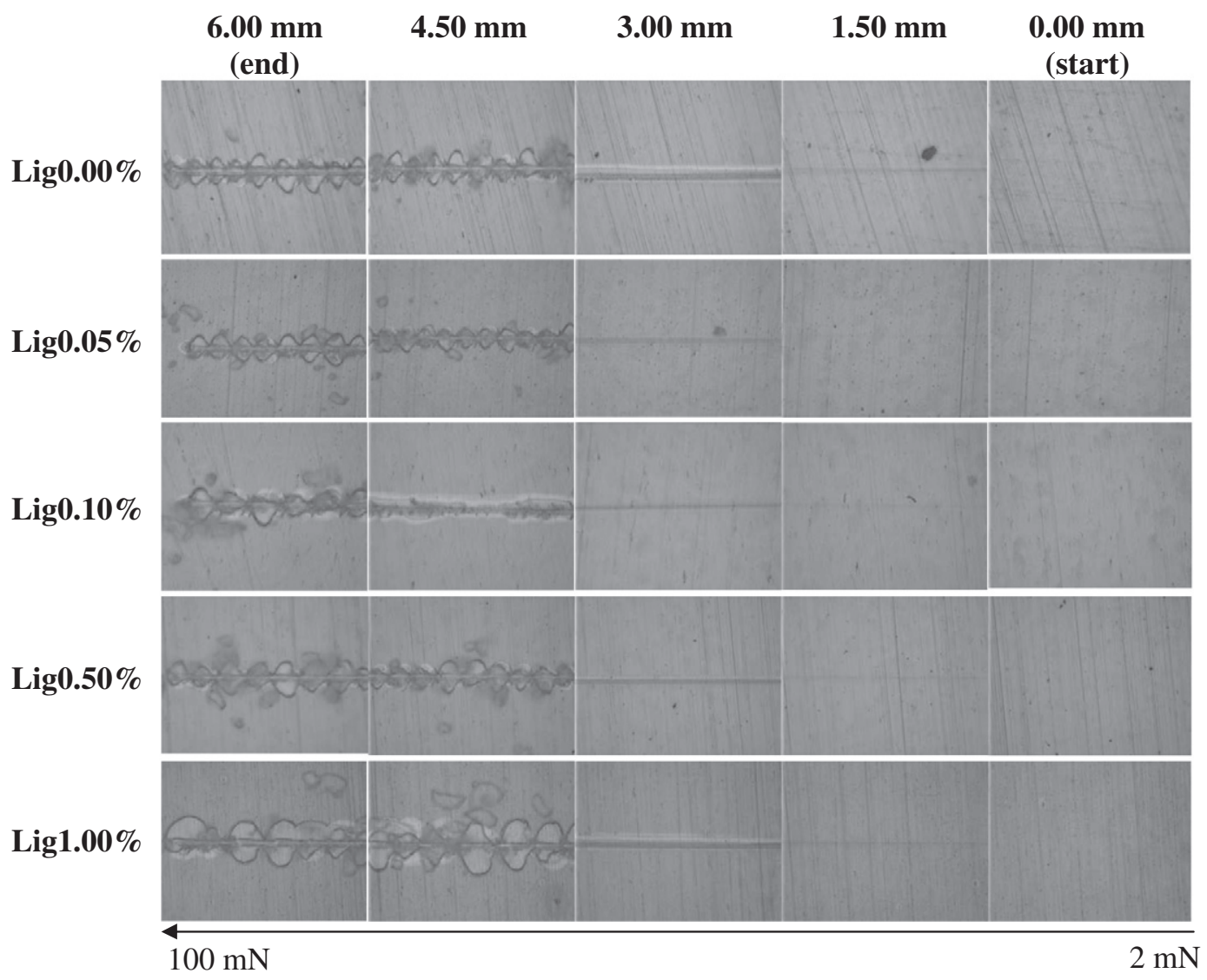

Fig. 10. Optical micrographs obtained after the scratch tests of siloxane-PMMA hybrid coating on carbon steel containing different quantities of lignin. 

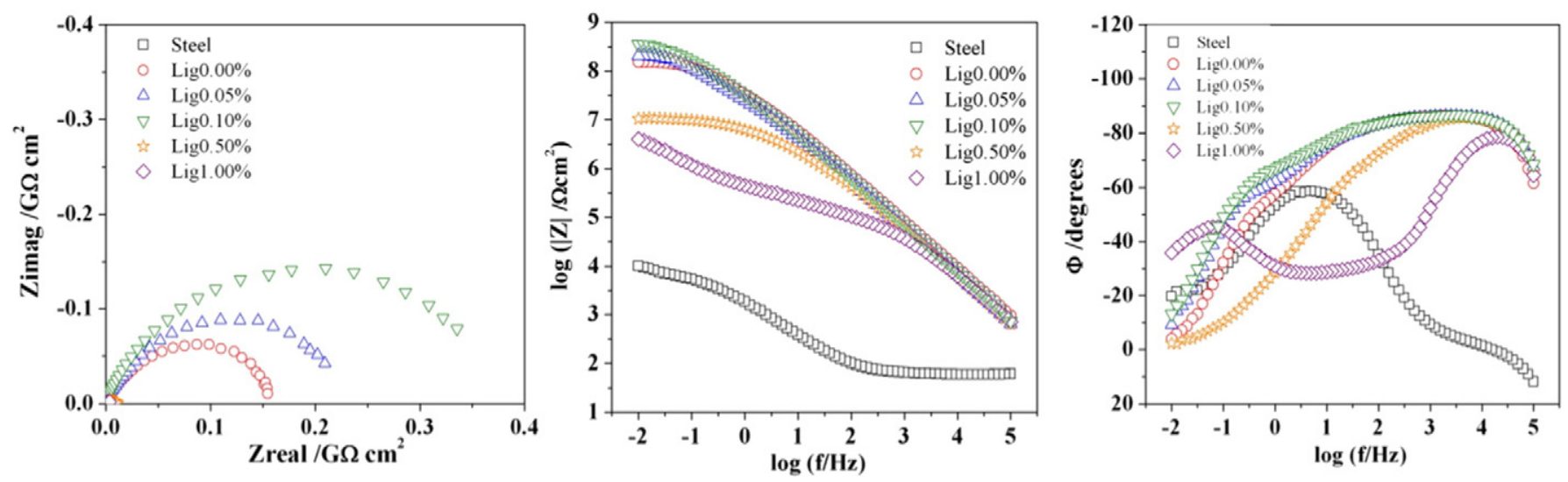

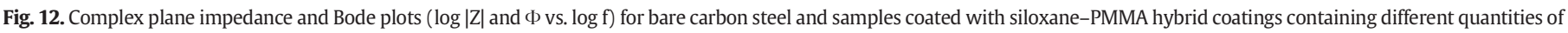
lignin after 1 day immersed in 3.5\% saline solution.

\subsection{Wettability and mechanical properties}

Contact angle measurements were undertaken to evaluate wettability of the coating surface. The higher the contact angle of water drops on the hybrid coating, more hydrophobic is the surface, being then less susceptible to wet corrosion. The measured contact angles of hybrids with different lignin loads are displayed in Fig. 7. In comparison with the bare steel, with contact angle of $71.8^{\circ}$, the hybrids are more hydrophobic. For carbon steel coated with pure hybrid a contact angle of $79.3^{\circ}$ was measured. Upon lignin addition the contact angle increased to $87.9^{\circ}$ for the Lig0.01\% sample and then decreased for higher concentrations. The increase of the contact angle, observed for low intermediate lignin concentrations, can be the result of polycondensation of lignin hydroxyl groups with those of the inorganic phase, during the sol-gel process. This leads to a decrease of the total amount of hydroxyl surface groups, and thus to a reduction of the polar nature of the hybrid, yielding more hydrophobic coatings. The addition of higher lignin amounts ( $0.50 \mathrm{wt}$.\% and 1.00 wt.\%) leads an excess of hydroxyl groups, responsible for the decrease of the hydrophobicity of the surface.

The results of microhardness measurements on monolith samples are displayed in Fig. 8. The siloxane-PMMA hybrid shows a microhardness of $22.9 \mathrm{HV}$, which is close to that of PMMA (22 HV) [33]. This was expected because the hybrid structure contains a higher percentage of organic than siloxane phase, which has a microhardness of about 300 HV [34]. Compared do the reference sample the hardness increased 35\% with increasing lignin loading up to $0.50 \mathrm{wt}$.\%, then remaining constant.
The coating thickness, determined with an optical system, increased slightly from 2.3-2.6 $\mu \mathrm{m}$ with the lignin content (Table 2). The values of the critical tensile pull-off force for detaching of the coatings are shown in Table 2. It can be observe that the detaching force varies between 3.4 MPa and 4.1 MPa, reaching the highest value for the Lig0.10\% sample.

These findings are in agreement with complementary results obtained for the adhesion of the coatings and its scratch resistance by microscratch measurements. The scratch tests provide the friction coefficient between the coating and the tip and critical load for delimitation of the coating. For the pure hybrid the friction coefficient increased with increasing load $(2-100 \mathrm{mN})$ from a value of 0.3 until reaching the steel substrate $(\mu=0.6)$, characterized by the noisy signal starting at a distance close to $3.50 \mathrm{~mm}$ (Fig. 9). The lignin addition did not change significantly the friction coefficient however it changed the necessary load to reach the substrate, observed by optical microscopy (Fig. 10). Optical microscopy images of the scratch track, shown in Fig. 10, were obtained for five regions of the scratch track: $0.0 \mathrm{~mm}$ (starting point), $1.5 \mathrm{~mm}, 3.0 \mathrm{~mm}, 4.5 \mathrm{~mm}$ and $6.0 \mathrm{~mm}$ (end point). All hybrids showed four stages of deformation with increasing force applied to the hybrid coating: (1) elastic deformation, (2) plastic deformation, (3) coating cracks and (4) delamination. The distance between the end point and the first point of delamination was referred as $l_{2}$, which was used to determine the critical force for delamination, shown in Fig. 11. The lignin-containing hybrids were harder, more scratch resistant and adherent than the pure hybrid matrix. The reinforcement induced by addition of $0.10 \mathrm{wt}$.\% of lignin in siloxane-PMMA hybrid is
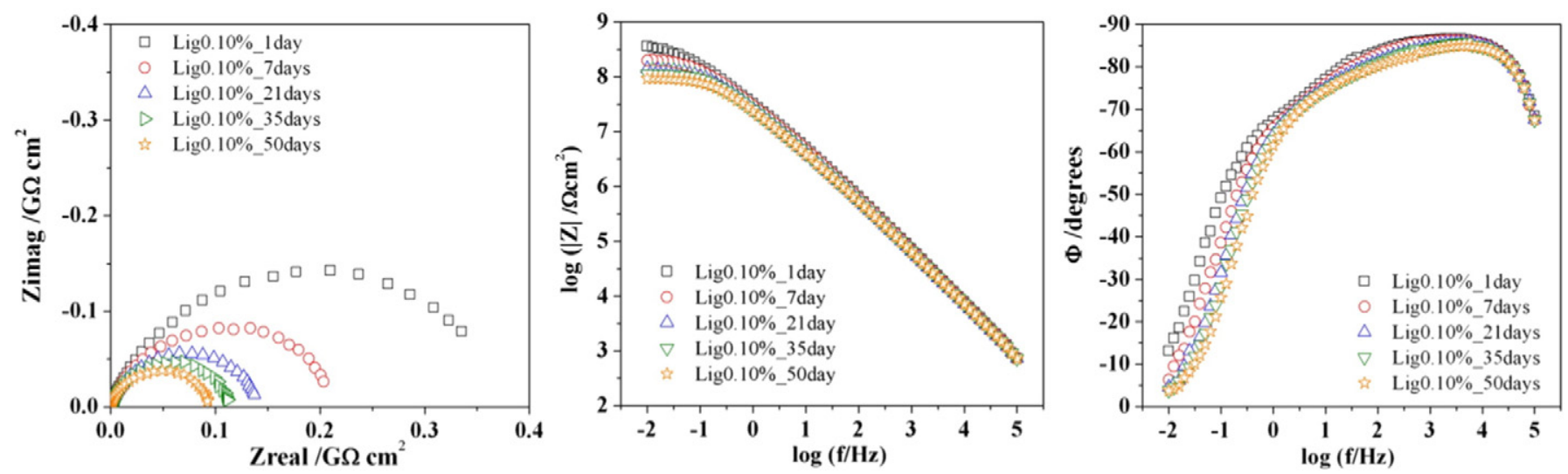

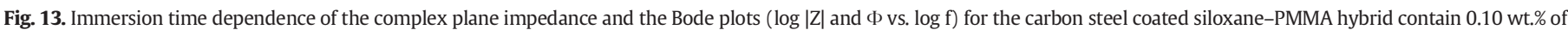
lignin in $3.5 \%$ saline solution. 
worth noting: compared with the Lig $0.00 \%$ sample, with a critical load of $55 \mathrm{mN}(3.26 \mathrm{~mm})$, the Lig0.10\% started to delaminate at about $80 \mathrm{mN}$ $(4.80 \mathrm{~mm})$, indicating a significant increase of the mechanical strength of the coating.

\subsection{Corrosion protection}

Fig. 12 displays the electrochemical impedance spectroscopy curves corresponding to carbon steel coated with lignin-containing hybrid recorded after one day of immersion in $3.5 \% \mathrm{NaCl}$ saline solution. All hybrid coated samples showed several orders of magnitude higher corrosion protection than bare carbon steel, most clearly visible on the frequency dependence of the impedance modulusIZI. Following sequence for increasing corrosion efficiency was established: $\operatorname{Lig} 0.10 \% \approx \operatorname{Lig} 0.00 \% \approx \operatorname{Lig} 0.05 \%>\operatorname{Lig} 0.50 \%>\operatorname{Lig} 1.00 \%$. The $\operatorname{Lig} 1.00 \%$ and Lig $0.50 \%$ hybrids presented clearly lower impedance values, with the appearance of a second time constant at low frequency, indicative of corrosion process at the coating/steel interface. Samples containing lower lignin concentrations (Lig0.00\%, Lig0.05\% and Lig0.10\%) presented an impedance modulus above $10^{8} \Omega \mathrm{cm}^{2}$, more than four orders of magnitude higher than that of bare carbon steel.

The phase angle at high frequency with values below $-80^{\circ}$ for at least three decades show a capacitive characteristic $\left(\Phi=-90^{\circ}\right)$, indicative for a high passivation capacity in blocking the electrolyte uptake of the coating. The samples were kept in saline solution and EIS measurements were performed every 7 days until the impedance modulus decreased by at least two orders of magnitude, associated with the appearance of micrometric pits. Again, the best performance was found for Lig $0.10 \%$ sample, which maintained high corrosion resistance $\left(>10^{8} \Omega \mathrm{cm}^{2}\right)$ for more than 50 days (Fig. 13) with an impedance modulus almost unchanged, showing a significant stability improvement compared to the previously reported siloxane-PMMA coatings [17]. These results highlight the Lig0.10\% hybrid with intermediate lignin content for which the excellent anti-corrosion performance can be directly related to the high contact angle, superior mechanical properties and good adhesion to the steel substrate. This performance of siloxanePMMA lignin hybrid blend coating is associated with its non-porous structure, formed by a dense and highly crosslinked hybrid network that acts as efficient diffusion barrier against corrosive species [17]. The silanol groups formed by hydrolysis of starting reagents TEOS and MPTS together with the hydroxyl groups of lignin anchor the coating on the metal through $\mathrm{Fe}-\mathrm{O}-\mathrm{Si}$ and $\mathrm{FeO} \cdots \mathrm{HOR}$ bonds [20], thus promoting a high adhesive strength between the coating and the substrate.

\section{Conclusions}

Lignin extracted from sugar cane bagasse (organosolv process) was added in different quantities to siloxane-PMMA hybrid synthesized from sol-gel process, and then easily deposited on carbon steel by dip-coating. The results indicated homogeneous dispersion of lignin in the hybrid matrix, thus yielding low-roughness coatings and increased hydrophobicity, hardness, adherence, scratch resistance and improved anti-corrosion resistance in saline environments. The time dependence of the electrochemical impedance showed that the hybrids with intermediate lignin quantity ( $0.10 \mathrm{wt} . \%)$ act as efficient diffusion barriers, with corrosion resistance of approximately $10^{8} \Omega \mathrm{cm}^{2}$ after long-term exposure in a $3.5 \% \mathrm{NaCl}$ aqueous solution. Lignin has proved to be a functional, environmentally friendly and low cost additive for the reinforcement of the siloxane-PMMA hybrid matrix.

\section{Acknowledgment}

The authors are thankful to the Metallic Materials Laboratory from Institute of Chemistry, UNESP, Brazil, for access to the microhardness apparatus and to John Nunn from the National Physical Laboratory, England, for the microscratch equipment. This work was supported by CNPq (309989/2012-4) and FAPESP (2013/11545-8).

\section{References}

[1] A. Heredia, A. Jiménez, R. Guillén, Z. Lebensm. Unters. Forsch. 200 (1995) 24-31.

[2] E. Dorrestijn, L.J.J. Laarhoven, I.W.C.E. Arends, P. Mulder, J. Anal. Appl. Pyrolysis 54 (2000) 153-192.

[3] R. Silva, K. Shirani, E.C. Haraguchi, F. Muniz, F.R. Adley, Quim. Nova 32 (2009) 661-671.

[4] C. Zhao, J.A. Lercher, Catalytic Depolymerization and Deoxygenation of Lignin, Ch 9: The Role of Catalysis for the Sustainable Production of Bio-fuels and Bio-Chemicals, Elsevier, Langford Lanes, BC, 2013. 290

[5] N. Hansjörg, S. Hinnerk, R. Mülhaupt, Macromol. Mater. Eng. 286 (2001) 737-743.

[6] C.G. Sánchez, L.A.E. Alvarez, Die Angew. Chem. 272 (1999) 65-70.

[7] P. Alexy, B. Kosiková, G. Podstránska, Polymer 41 (2000) 4901-4908.

[8] G. Milczarek, Electrochim. Acta 54 (2009) 3199-3205.

[9] W. Hoareau, F.B. Oliveira, S. Grelier, B. Siegmund, E. Frollini, A. Castellan, Macromol Mater. Eng. 291 (2006) 829-839.

[10] M.N. Vanderlaan, R.W. Thring, Biomass Bioenergy 14 (1998) 525-529.

[11] N.S. Çetin, Int. J. Adhes. 22 (2002) 481-486.

[12] R.J.A. Gosselink, M.H.B. Snijder, A. Kranenbarg, E.R.P. Keijsers, E. de Jong, L.L. Sigsson, Ind. Crop. Prod. 20 (2004) 191-203.

[13] N.M. Monteiro, V. Calado, R.J.S. Rodriguez, F.M. Margem, J. Mater. Res. Technol. 1 (2012) 117-126.

[14] F. Mammeri, L. Rozes, C. Sanchez, E. Le Bourhis, J. Sol-Gel Sci. Technol. 26 (2003) 413-417.

[15] F. Mammeri, L. Rozes, E.L. Bourhis, C. Sanchez, J. Eur. Ceram. Soc. 26 (2006) 267-272

[16] V.H.V. Sarmento, M.G. Schiavetto, P. Hammer, A.V. Benedetti, C.S. Fugivara, P.H Suegama, S.H. Pulcinelli, C.V. Santilli, Surf. Coat. Technol. 204 (2010) 2689-2701.

[17] P. Hammer, F.C. dos Santos, B.M. Cerrutti, S.H. Pulcinelli, C.V. Santilli, J. Sol-Gel Sci. Technol. 63 (2012) 266-274.

[18] P. Hammer, M.G. Schiavetto, F.C. dos Santos, A.V. Benedetti, S.H. Pulcinelli, C.V Santilli, Non-Cryst. Solids 356 (2010) 2606-2612.

[19] P. Hammer, F.C. dos Santos, B.M. Cerrutti, S.H. Pulcinelli, C.V. Santilli, Prog. Org. Coat 76 (2013) 601-608.

[20] S.V. Harb, F.C. dos Santos, B.L. Caetano, S.H. Pulcinelli, C.V. Santilli, P. Hammer, RSC Adv. 5 (2015) 15414-15424.

[21] B. Balgude, A. Sabnis, J. Sol-Gel Technol. 64 (2012) 124-134.

[22] J.L. Oliverio, A.G.P. Hilst, Int. Sugar J. 106 (2004) 169-171.

[23] DEDINI Indústrias de Base (Piracicaba, SP). Antonio Geraldo Proença Hilst Aperfeiçoamento em Processo de Hidrólise Ácida Rápida de Material Lignocelulósico e em Reator de Hidrólise. Brasil patente BR n. PI 0505212-2A, 2005 November 01, 2007 August 07.

[24] B.M. Cerrutti, C.S. de Souza, A. Castellan, R. Ruggiero, E. Frollini, Ind. Crop. Prod. 36 (2012) 108-115.

[25] W.D. Callister Jr., Materials: Mechanical Properties, Ch 06: Materials Science and Engineering: An Introduction, John Wiley \& Sons, New York, 2008. 149.

[26] C. Pouteau, S. Baumberger, B. Cathala, P. Dole, Biologies 327 (2004) 935-943.

[27] A. Streitwieser, C.H. Heathcock, E.M. Kosower, Ch 30: Introduction to Organic Chemistry, second ed. Macmillan, New York, 1981. 995.

[28] A.V. Naumkin, A. Kraut-Vass, S.W. Gaarenstroom, C.J. Powell, J. Nist, X-ray Photoelectron Spectroscopy Database, NIST Standard Reference Database 20, Version 4.1. Created June 06 2000. Last updated 15 2012. Available from http://srdata.nist.gov/XPS/.

[29] Y.T. Wang, T.C. Chang, Y.S. Hong, H.B. Chen, Thermochim. Acta 397 (2003) 219-226

[30] T. Kashiwagi, A. Inaba, J.E. Brown, K. Hatada, T. Kitayama, E. Masuda, Macromolecules 19 (1986) 2160-2168.

[31] C.J.T. Landry, B.K. Coltrain, J.A. Wesson, N. Zumbulyadis, J.L. Lippert, Polymer 33 (1992) 1496-1506.

[32] T. Dizhbite, G. Telysheva, V. Jurkjane, U. Viesturs, Bioresour. Technol. 95 (2004) 309-317.

[33] M. Okamoto, J. Appl. Polym. Sci. 83 (2002) 2774-2779.

[34] O. de Sanctis, L. Gomez, N. Pellegri, C. Parodi, A. Marajofsky, A. Duran, J. Non-Cryst. Solids 121 (1990) 338-343. 